\title{
NONCLOSED PURE SUBGROUPS OF LOCALLY COMPACT ABELIAN GROUPS
}

\author{
YUJI TAKAHASHI \\ (Communicated by Jonathan M. Rosenberg) \\ Dedicated to Professor Shozo Koshi on his sixtieth birthday
}

\begin{abstract}
We study the existence of many nonclosed pure subgroups of nondiscrete locally compact abelian groups. It is shown that every nondiscrete locally compact abelian group has uncountably many nonclosed pure subgroups. This in particular solves a question of Armacost. It is also shown that, if $G$ is a nondiscrete locally compact abelian group and if either $G$ is a compact group or the torsion part $t(G)$ of $G$ is nonopen, then $G$ has $2^{c}$ proper dense pure subgroups, where $c$ denotes the power of the continuum. This in particular gives a partial answer to another question of Armacost.
\end{abstract}

It is well-known that if a locally compact (Hausdorff) abelian group has no nonclosed subgroups, then it is discrete (see, e.g., $[1,1.26])$. In connection with this fact, Armacost [1, 7.24] asked whether every locally compact abelian group with no nonclosed pure subgroups must be necessarily discrete. Recall that a subgroup $H$ of an abelian group $G$ is called pure in $G$ (or a pure subgroup of $G$ ) if $n H=H \cap n G$ for every integer $n$. (For an abelian group $G$ and an integer $n, n G$ denotes the subgroup $\{n x: x \in G\}$ of $G$.) In this note we first show that Armacost's question has an affirmative answer. We indeed prove the following stronger result.

Theorem 1. Every nondiscrete locally compact abelian group $G$ has uncountably many nonclosed pure subgroups.

Note that there exist nondiscrete locally compact abelian groups which contain no proper closed pure subgroups (cf. [1, Proposition 7.11]).

In [8] it was observed that not every nondiscrete locally compact abelian group has a proper dense subgroup. Later, Khan [6, Proposition 5.1] proved that a nondiscrete locally compact abelian group $G$ has no proper dense subgroups if and only if $t(G)$ (the maximal torsion subgroup of $G$ ) is open and $p G$ is open for every prime $p$. (See also [5]. For some related results, see [1, 2.26], [2,

Received by the editors August 7, 1989 and, in revised form, November 2, 1989.

1980 Mathematics Subject Classification (1985 Revision). Primary 22B05, 22D05.

Key words and phrases. Locally compact abelian groups, pure subgroups, nonclosed subgroups, dense subgroups. 
6-8, 10].) In conjunction with these results, the following question was raised in $[1,7.18(\mathrm{c})]$ : Which locally compact abelian groups have no proper dense pure subgroups? We prove the following theorem as a partial answer to this second question of Armacost.

Theorem 2. Let $G$ be a nondiscrete locally compact abelian group. If either $G$ is a compact group or $t(G)$ is nonopen, then $G$ has $2^{c}$ proper dense pure subgroups, where $c$ denotes the power of the continuum.

Armacost shows that if a locally compact abelian group $G$ is not totally disconnected, then $G$ contains at least one proper dense pure subgroup [1, 7.18(b)]. Note that, if a locally compact abelian group $G$ is not totally disconnected, then $t(G)$ is nonopen (see, e.g., [4, Theorem (24.21)]). It is also easy to see that there exist totally disconnected locally compact abelian groups $G$ such that $t(G)$ are nonopen. For example, the $p$-adic integers $J_{p}$ and the $p$-adic numbers $F_{p}$ for every prime $p$ are nondiscrete totally disconnected torsion-free groups and hence $t\left(J_{p}\right)$ and $t\left(F_{p}\right)$ are nonopen. Thus Theorem 2 strengthens and improves the Armacost's result mentioned above.

We now turn to the proof of Theorem 1. Recall the following result, which is fundamental in studying pure subgroups of an abelian group and which plays an important role in proving our Theorem 1 . The proof is simple and is to be found in [3, Proposition 26.2].

Lemma 1. Let $G$ be an abelian group, and let $K$ be a countably infinite subgroup of $G$. Then there exists a pure subgroup $H$ of $G$ such that $H$ is countably infinite and contains $K$.

To prove Theorem 1, we need another simple lemma, which is essential in our constructions of nonclosed subgroups.

Lemma 2. Let $G$ be a nondiscrete locally compact abelian group and let $x$ be an element of $G$. Then there exists a sequence $\left\{x_{n}\right\}_{n=1}^{\infty}$ of distinct elements $x_{n}$ which converges to $x$ as $n \rightarrow \infty$.

Proof. We may assume that $x=0$ (the identity element of $G$ ). It also suffices to show that $G$ has a closed metrizable subgroup $H$ which is nondiscrete. By the structure theorem of locally compact abelian groups, there exist a nonnegative integer $n$ and a locally compact abelian group $G_{0}$ such that $G$ is topologically isomorphic with $\mathbf{R}^{n} \times G_{0}$ and $G_{0}$ contains a compact open subgroup $K$ [4, Theorem (24.30)]. If $n \neq 0$, then we have only to take $H=\mathbf{R}^{n}$. If $n=0$, then $G=G_{0}$ and $K$ is infinite because $G$ is nondiscrete. Thus it follows from [9, Theorem 2.5.2] that $K$ has an infinite compact (and hence nondiscrete) metrizable subgroup $H$.

Proof of Theorem 1. We use the symbol $\omega_{1}$ to denote the smallest uncountable ordinal number. We proceed by transfinite induction to construct countably infinite pure subgroups $H_{\alpha}$ for all $\alpha$ with $\alpha<\omega_{1}$. Let us make use of Lemma 2 to choose a sequence $\left\{x_{n}\right\}_{n=1}^{\infty}$ in $G$ such that $\left\{x_{n}\right\}_{n=1}^{\infty}$ converges to 0 as 
$n \rightarrow \infty$ and consists of distinct elements of $G$. Let $\alpha_{0}$ denote the minimum element of the set $\left\{\alpha: \alpha<\omega_{1}\right\}$ of all ordinal numbers less than $\omega_{1}$ and let $K_{\alpha_{0}}$ be the subgroup generated by $\left\{x_{n}\right\}_{n=1}^{\infty}$. Then $K_{\alpha_{0}}$ is countably infinite, and hence Lemma 1 implies that there exists a pure subgroup $H_{\alpha_{0}}$ of $G$ such that $H_{\alpha_{0}}$ is countably infinite and contains $K_{\alpha_{0}}$. Now suppose that $\beta<\omega_{1}$ and that the countably infinite pure subgroups $H_{\alpha}$ of $G$ have been defined for all $\alpha<\beta$. Let $L_{\beta}$ denote the subgroup generated by the union $\cup\left\{H_{\alpha}: \alpha<\beta\right\}$. Since $\{\alpha: \alpha<\beta\}$ is at most countably infinite and $H_{\alpha}$ is countably infinite for every $\alpha$ with $\alpha<\beta$, the union $\cup\left\{H_{\alpha}: \alpha<\beta\right\}$ is countably infinite and therefore $L_{\beta}$ is too. Thus we can select an element $y_{\beta}$ of $G$ which is not contained in $L_{\beta}$, because $G$ is nondiscrete and hence has cardinal number greater than or equal to $c[4,(4.26)]$. Let $K_{\beta}$ denote the subgroup generated by $L_{\beta}$ and $y_{\beta}$. Then, by Lemma 1 , there exists a pure subgroup $H_{\beta}$ of $G$ such that $H_{\beta}$ is countably infinite and contains $K_{\beta}$, which completes our transfinite induction. It is clear from the definition of $\left\{H_{\alpha}: \alpha<\omega_{1}\right\}$ that $H_{\alpha} \varsubsetneqq H_{\beta}$ for every $\alpha$ and $\beta$ with $\alpha<\beta<\omega_{1}$. It remains only to show that $H_{\alpha}$ is nonclosed in $G$ for every $\alpha$ with $\alpha<\omega_{1}$. To see this, suppose that $H_{\alpha}$ is closed for some $\alpha$. Since $H_{\alpha}$ is a countably infinite locally compact abelian group, the Baire category theorem and [4, Theorem (5.8)] imply that every element of $H_{\alpha}$ is an isolated point. But this is a contradiction because 0 is not an isolated point. This completes the proof of Theorem 1 .

We now proceed to the proof of Theorem 2 . The following fact is easily seen by a routine argument, so we omit the proof.

Lemma 3. Let $G$ be a locally compact abelian group and let $K$ be a proper pure subgroup of $G$. Let $\pi$ denote the natural homomorphism from $G$ onto $G / K$. Then we have:

(1) If $K$ is closed and if $\widetilde{H}$ is a proper dense pure subgroup of $G / K$, then $H=\pi^{-1}(\widetilde{H})$ is a proper dense pure subgroup of $G$.

(2) If $K$ is dense in $G$ and if $\widetilde{H}$ is a proper pure subgroup of $G / K$, then $H=\pi^{-1}(\widetilde{H})$ is a proper dense pure subgroup of $G$.

Proof of Theorem 2. We first consider the case where $t(G)$ is nonopen. Note that their proof of [10, Theorem 2.5$]$ shows the following: Every locally compact abelian group whose torsion part $t(G)$ is nonopen has a proper dense subgroup $K$ such that $G / K$ is algebraically isomorphic with $\mathbf{T}$ (the circle group). We now claim that there exists a proper subgroup $H$ of $G$ such that $H$ contains $K, G / H$ is torsion-free, and the torsion-free rank $r_{0}(G / H)$ of $G / H$ is equal to $c$. To see this, let us take $H=\pi^{-1}(t(G / K))$, where $\pi$ denotes the natural homomorphism from $G$ onto $G / K$. Then $H$ is a subgroup with the desired property. It is clear that $H$ is a proper subgroup of $G$ containing $K$. Note that $G / H$ is algebraically isomorphic with $(G / K) / t(G / K)$ and therefore with $\mathbf{T} / t(\mathbf{T})$. Thus $G / H$ is torsion-free and $r_{0}(G / H)=r_{0}(\mathbf{T} / t(\mathbf{T}))=c$. This completes the proof of our claim. Since $H$ includes $K, H$ is dense in $G$. Note 
that $H$ is pure in $G$ because $G / H$ is torsion-free. Since $r_{0}(G / H)=c, G / H$ has $2^{c}$ neat subgroups $\left\{\widetilde{H}_{\alpha}\right\}$ [3, $\S 31$, Exercise 18]. (Recall that a subgroup $B$ of an abelian group $A$ is called a neat subgroup of $A$ if $p B=B \cap p A$ for every prime $p$.) As is easily verified, neatness and purity are equivalent in torsion-free groups. Hence each neat subgroup $\widetilde{H}_{\alpha}$ is pure in $G / H$. Now let $H_{\alpha}=\rho^{-1}\left(\widetilde{H}_{\alpha}\right)$ for each $\alpha$, where $\rho$ denotes the natural homomorphism from $G$ onto $G / H$. Then Lemma 3 implies that $H_{\alpha}$ is a proper dense pure subgroup of $G$ for each $\alpha$. Thus we conclude that $G$ has $2^{c}$ proper dense pure subgroups.

Next suppose that $G$ is compact. As we have already seen, $G$ has $2^{c}$ proper dense pure subgroups if $t(G)$ is nonopen. Thus we may assume that $t(G)$ is open. Then it follows from [4, Theorem (25.9), $(25.30(\mathrm{~b}))$, and (A.25)] that there exist a prime $p$ and a positive integer $r$ such that $G$ is topologically isomorphic with the direct product of $G_{1}=\prod \mathbf{Z}\left(p^{r}\right)$ (the direct product of countably infinite many copies of the finite cyclic group $\mathbf{Z}\left(p^{r}\right)$ of order $\left.p^{r}\right)$ and a compact abelian group $G_{2}$ of bounded order. Let $K_{1}$ denote the weak direct product of all components of $G_{1}$. Then, of course, $K_{1}$ is a countably infinite dense pure subgroup of $G_{1}$. It is also clear that $K_{1} \times G_{2}$ is a dense pure subgroup of $G_{1} \times G_{2}$ and that $\left(G_{1} \times G_{2}\right) /\left(K_{1} \times G_{2}\right)$ is a $p$-group of bounded order. Since $G_{1}$ has cardinal number equal to $c, G_{1} / K_{1}$ and hence $\left(G_{1} \times G_{2}\right) /\left(K_{1} \times G_{2}\right)$, also, have cardinal number $c$. Thus we conclude that there exist a prime $p$ and a proper dense pure subgroup $K$ of $G$ such that $G / K$ is a $p$-group of bounded order and has cardinal number equal to $c$. Let $\widetilde{E}$ be a maximal pure independent subset of $G / K$ and let $\widetilde{F}$ denote the subgroup of $G / K$ generated by $\widetilde{E}$. Then $(G / K) / \widetilde{F}$ is divisible ([3; $\S 33$, Exercise 1$]$ or [4, (A.24)]). Since $G / K$ has bounded order, $\widetilde{F}$ coincides with $G / K$. Thus $\widetilde{E}$ has cardinal number equal to $c$. Let $\left\{\widetilde{E}_{\alpha}\right\}$ denote the family of all proper subsets of $\widetilde{E}$, and let $\widetilde{H}_{\alpha}$ denote the subgroup of $G / K$ generated by $\widetilde{E}_{\alpha}$ for each $\alpha$. Then, of course, $\left\{\widetilde{E}_{\alpha}\right\}$ has cardinal number equal to $2^{c}$. It is also easy to see that each $\widetilde{H}_{\alpha}$ is a proper pure subgroup of $G / K$ and that $\left\{\widetilde{H}_{\alpha}\right\}$ has cardinal number equal to $2^{c}$. Now Lemma 3 implies that $G$ has $2^{c}$ proper dense pure subgroups. This completes the proof of Theorem 2 .

Remarks. 1. The cardinal number $2^{c}$ in Theorem 2 is best possible in the sense that there exist nondiscrete locally compact abelian groups which contain exactly $2^{c}$ proper dense pure subgroups. It is easy to see that $\mathbf{R}$ and $\mathbf{T}$ have exactly $2^{c}$ proper dense pure subgroups.

2. In [6, Theorem 5.1] Khan proved that the nonclosed subgroups of a nondiscrete locally compact abelian group do not satisfy the maximum condition. The proof of our Theorem 1 improves Khan's result: The nonclosed pure subgroups of a nondiscrete locally compact abelian group do not satisfy the maximum condition. Khan [6, Lemma 5.1 and Theorem 5.2] also proved that if $G$ is a nondiscrete locally compact abelian group and if either $G$ is a compact group 
or $t(G)$ is nonopen, then the proper dense subgroups of $G$ satisfy neither the maximum condition nor the minimum condition. From the proof of Theorem 2 we can easily show an improvement of this Khan's result: If $G$ is a nondiscrete locally compact abelian group and if either $G$ is a compact group or $t(G)$ is nonopen, then the proper dense pure subgroups of $G$ satisfy neither the maximum condition nor the minimum condition.

\section{ACKNOWLEDGMENT}

The author is grateful to the referee for useful remarks, and for drawing his attention to [5].

\section{REFERENCES}

1. D. L. Armacost, The structure of locally compact abelian groups, Marcel Dekker, New York, 1981.

2. W. E. Dietrich, Jr., Dense decompositions of locally compact groups, Colloq. Math. 24 (1972), 147-151.

3. L. Fuchs, Infinite abelian groups, vol. I, Academic Press, New York, 1970.

4. E. Hewitt and K. Ross, Abstract harmonic analysis, vol. I, Springer-Verlag, Berlin, Heidelberg, New York, 1963.

5. M. I. Kabenjuk, Dense subgroups of locally compact abelian groups, Sibirsk. Mat. Zh. 21 (1980), 202-203.

6. M. A. Khan, Chain conditions on subgroups of LCA groups, Pacific J. Math. 86 (1980), 517-534.

7. $\ldots$ When are proper subgroups of LCA groups contained in maximal ones?, Proc. Amer. Math. Soc. 83 (1981), 131-137.

8. M. Rajagopalan and H. Subrahmanian, Dense subgroups of locally compact groups, Colloq Math. 35 (1976), 289-292.

9. W. Rudin, Fourier analysis on groups, Interscience, New York, 1962.

10. S. Saeki and K. Stromberg, Measurable subgroups and nonmeasurable characters, Math. Scand. 57 (1985), 359-374.

Department of Mathematics, Kushiro Public University of Economics, 4-1-1 Ashino, KUSHIRO, 085 JAPAN 